\title{
HERMITIAN SPACES IN GEODESIC CORRESPONDENCE ${ }^{1}$
}

\author{
W. J. WESTLAKE
}

1. Coburn [1] has studied the problem of Hermitian spaces in geodesic correspondence. He found a necessary and sufficient condition for two Kähler spaces to be in geodesic correspondence and showed that such correspondence was impossible between a Kähler space and a Hermitian space. The problem of geodesic correspondence between two Hermitian spaces he left unsolved.

It will be shown that Coburn's first result is incorrect, as was pointed out by Bochner [2]; and we shall solve the general problem, giving necessary and sufficient conditions for two Hermitian spaces to be in geodesic correspondence.

2. The linear connection of a Hermitian space, $H_{n}$, is defined to be

$$
\Gamma_{\beta \gamma}^{\alpha}=g^{\alpha \lambda^{*}} \partial_{\gamma} g_{\beta \lambda^{*}} .
$$

We use the usual convention of Hermitian geometry that Greek indices run from 1 to $n$ and Latin indices from 1 to $2 n$ where $\alpha^{*}=\alpha+n$.

The torsion tensor $S_{j k}^{b}$ is defined to be

$$
S_{j k}^{i}=\Gamma_{[j k]}^{i}
$$

and its vanishing characterises a Kähler space, $K_{n}$. We shall also introduce a connection $E_{j k}^{i}$ which is analogous to the Christoffel symbol

$$
\left\{\begin{array}{c}
i \\
j k
\end{array}\right\}
$$

of Riemannian geometry. This is:

$$
E_{j k}^{i}=\frac{1}{2} g^{i h}\left(\partial_{k} g_{j h}+\partial_{j} g_{k h}-\partial_{h} g_{j k}\right)
$$

and may also be written in the form:

$$
E_{j k}^{i}=\Gamma_{(j k)}^{i}+g^{i p} g_{j q} S_{p k}^{q}+g^{i p} g_{k q} S_{p j}^{q} .
$$

For a Kähler space, it is clear that $E_{j k}^{\mathfrak{t}}$ reduces to $\Gamma_{j k}^{\ell}$.

Received by the editors June 30, 1953.

1 This paper is a short extract from a thesis approved by the University of London for the degree of Ph.D. It was written while the author was the recipient of a D.S.I.R. research grant. 
The differential equations of the geodesics of a Hermitian space (given by Coburn [1]) may be rewritten in terms of $E_{j k}^{i}$ and are:

$$
\frac{d^{2} z^{i}}{d t^{2}}+E_{j k}^{i} \frac{d z^{i}}{d t} \frac{d z^{k}}{d t}-\frac{d z^{i}}{d t}\left(\frac{d^{2} s}{d t^{2}} / \frac{d s}{d t}\right)=0
$$

with $t$ as parameter. Multiplying by $d z^{m} / d t$, interchanging $i$ and $m$, and subtracting, we have the alternative form:

$$
\left(\frac{d^{2} z^{i}}{d t^{2}} \frac{d z^{m}}{d t}-\frac{d^{2} z^{m}}{d t^{2}} \frac{d z^{i}}{d t}\right)+\left(E_{j k}^{i} \frac{d z^{m}}{d t}-E_{j k}^{m} \frac{d z^{i}}{d t}\right) \frac{d z^{j}}{d t} \frac{d z^{k}}{d t}=0
$$

3. Consider two Hermitian spaces $H_{n}, H_{n}^{\prime}$ whose geodesics correspond. The respective differential equations of their geodesics will be satisfied by the same functions $z^{i}(t)$ and subtracting we shall have:

$$
\left(A_{j k}^{i} \delta_{n}^{m}-A_{j k}^{m} \delta_{n}^{i}\right) \frac{d z^{n}}{d t} \frac{d z^{i}}{d t} \frac{d z^{k}}{d t}=0
$$

where the symmetric tensor $A_{j k}^{i}$ is defined by

$$
A_{j k}^{i}=E_{j k}^{\prime i}-E_{j k}^{i}
$$

and primed expressions refer to $H_{n}^{\prime}$. Equation (3.2) must be satisfied by arbitrary values of $d_{t} z^{i}$ at any point. Therefore we have:

$$
A_{\left(j k \delta_{n)}\right.}^{i}-A_{\left(j k \delta_{n)}\right.}^{m}=0
$$

and by virtue of the symmetry of $A_{j k}^{i}$, this may be written:

$$
A_{j k}^{i} \delta_{n}^{m}+A_{k n}^{i} \delta_{j}^{m}+A_{n j}^{i} \delta_{k}^{m}=A_{j k}^{m} \delta_{n}^{i}+A_{k n}^{m} \delta_{j}^{i}+A_{n j}^{m} \delta_{k}^{i} .
$$

4. If both spaces are Kähler, $A_{j k}^{i}$ has all its components identically zero except those of the type $A_{\beta_{\gamma}}^{\alpha}, A_{\beta^{*} \gamma^{*}}^{\alpha^{*}}$. Putting $i=\alpha, j=\beta, k=\gamma$, $m=\mu^{*}, n=\nu^{*}$ in (3.4), we see that

$$
A_{\beta \gamma}^{\alpha}=0 .
$$

And conversely if (4.1) holds, (3.1) is satisfied identically. But

$$
A_{\beta \gamma}^{\alpha}=\Gamma_{\beta \gamma}^{\prime \alpha}-\Gamma_{\beta \gamma}^{\alpha}
$$

and thus:

A necessary and sufficient condition for two Kähler spaces, $K_{n}, K_{n}^{\prime}$, to be in geodesic correspondence is that

$$
\Gamma_{\beta \gamma}^{\prime \alpha}=\Gamma_{\beta \gamma}^{\alpha} .
$$


This is the correction of Coburn's result referred to.

5. Suppose now that both spaces are Hermitian. Then equation (3.4) must again be satisfied. Putting $i=\alpha, j=\beta, k=\gamma, m=\mu^{*}$, $n=\nu^{*}$, we have

$$
A_{\beta \gamma}^{\alpha} \delta_{\nu^{*}}^{\mu^{*}}=A_{\gamma \nu^{*} \delta_{\beta}}^{\mu^{*}}+A_{\nu^{*} \beta}^{\mu^{*} \delta_{\gamma}^{*}}=0 \text {. }
$$

The only components of $A_{j k}^{i}$ that now vanish identically are those of the type $A_{\beta^{*} \gamma^{*}}^{\alpha}, A_{\beta \gamma}^{\alpha^{*}}$. In particular, $A_{\gamma v^{*}}^{\mu^{*}}$ is a tensor; and the contraction $A_{\gamma \mu^{*}}^{\mu^{*}}$, which we denote by $A_{\gamma}$, is a vector. Contracting on $\mu^{*}, \nu^{*}$ in (5.1), we have:

$$
n A_{\beta \gamma}^{\alpha}=A_{\gamma} \delta_{\beta}^{\alpha}+A_{\beta} \delta_{\gamma}^{\alpha} .
$$

This constitutes our first necessary condition.

In (3.4), putting $i=\alpha, j=\beta^{*}, k=\gamma, m=\mu, n=\nu$, and contracting on $\mu, \nu$ we have:

$$
n A_{\beta^{*} \gamma}^{\alpha}=A_{\beta^{*}}^{\alpha} \delta_{\gamma}^{\alpha},
$$

and this is our second necessary condition.

If now we assume that both these necessary conditions are satisfied, it is easy to verify that (3.4) is then identically satisfied for every possible combination of the indices, $i, j, k, m, n$. Thus (3.1) is identically satisfied and the two necessary conditions are also sufficient. Simplifying (5.2) and (5.3) and denoting by $S_{\alpha}$ the contraction $S_{\alpha \mu}^{\mu}$, our final result is:

The necessary and sufficient conditions for two Hermitian spaces $H_{n}, H_{n}^{\prime}$ to be in geodesic correspondence are:

$$
\begin{gathered}
n \Gamma_{(\beta \gamma)}^{\prime \alpha}+\delta_{(\beta)}^{\alpha} S_{\gamma)}^{\prime}=n \Gamma_{(\beta \gamma)}^{\alpha}+\delta_{(\beta)}^{\alpha} S_{\gamma)}, \\
n g^{\prime \alpha \lambda^{*}} g_{\gamma \nu^{*}}^{\prime} S_{\lambda^{*} \beta^{*}}^{\prime \nu^{*}}+\delta_{\gamma}^{\alpha} S_{\beta^{*}}^{\prime}=n g^{\alpha \lambda^{*}} g_{\gamma \nu^{*}} S_{\lambda^{*} \beta^{*}}^{\nu^{*}}+\delta_{\gamma}^{\alpha} S_{\beta^{*}}^{\alpha} .
\end{gathered}
$$

BiBLIOGRAPHY

1. N. Coburn, Unitary spaces with corresponding geodesics, Bull. Amer. Math. Soc. vol. 47 (1941) pp. 901-910.

2. S. Bochner, Curvature in Hermitian metric, Bull. Amer. Math. Soc. vol. 53 (1947) pp. 179-195.

UNIVERSITY OF SOUTHAMPTON 